\title{
Efeitos do Uso do Computador na Cognição, Estado Emocional, Qualidade de Vida e Habilidade Manual de Idosos
}

\author{
Michele Marinho da Silveira, ${ }^{1, *} \&$ Mirna Wetters Portuguez ${ }^{2}$ \\ ${ }^{1}$ Faculdade Meridional, Passo Fundo, RS, Brasil \\ ${ }^{2}$ Pontifícia Universidade Católica do Rio Grande do Sul, Porto Alegre, RS, Brasil
}

\begin{abstract}
RESUMO - Este estudo buscou verificar os efeitos do uso do computador no desempenho cognitivo, estado emocional, qualidade de vida e habilidade motora manual de participantes de oficinas de informática. A amostra foi constituída por 114 idosos, divididos em grupo sem informática (GSI) com 58 idosos e grupo informática (GI) com 56 idosos. Os instrumentos utilizados foram: Exame Cognitivo de Addenbrooke Revisado (ACE-R), Escala de Depressão Geriátrica (GDS-15), Inventário de Ansiedade de Beck (BAI), qualidade de vida (WHOQOL-Bref), força de preensão manual (dinamômetro Saehan Corporation ${ }^{\circledR}$ ) e motricidade fina (painel de habilidades manuais). Os resultados revelaram que o uso do computador contribuiu positivamente para a funcionalidade cognitiva do idoso, melhorando seu estado emocional, sua qualidade de vida e sua habilidade motora manual.
\end{abstract}

PALAVRAS-CHAVE: computador, cognição, destreza da mão, qualidade de vida, idoso

\section{Effects of Computer Use on Cognition, Emotional State, Quality of Life and Motor Skills of the Elderly}

\begin{abstract}
This study aimed to analyze the effects of computer use on the cognitive performance, emotional state, quality of life and motor skills of participants enrolled in computer classes. The sample consisted of 114 elderly people who were divided into two groups: one without computer skills (GSI) comprised of 58 people, and another with computer skills (CG), comprised of 56. For this study, we used Addenbrooke's Cognitive Examination-Revised (ACE-R), the Geriatric Depression Scale (GDS-15), the Beck Anxiety Inventory (BAI), a quality of life assessment (WHOQOL-Bref), and measurements of handgrip strength (dynamometer Saehan Corporation $\AA$ ) and fine motor skill (manual skills panel). The results showed that computer use may contribute positively to the cognitive function of the elderly, improving their emotional state, quality of life and motor skills.
\end{abstract}

KEYWORDS: computer, cognition, motor skills, quality of life, elderly

O processo de envelhecimento é visto de formas diferentes, alguns o caracterizam como uma diminuição geral das capacidades da vida diária, outros o consideram como um período de crescente vulnerabilidade e de cada vez maior dependência no seio familiar. Outros, ainda, veneram a velhice como o ponto mais alto da sabedoria, bom senso e serenidade (Fechine \& Trompieri, 2012).

Entretanto, ocorrem mudanças complexas que envolvem os aspectos físicos, sensoriais e cognitivos (Argimon \& Stein, 2005). As mudanças físicas interferem na coordenação motora, nos reflexos proprioceptivos, na flexibilidade, na força, na velocidade dos movimentos e na resistência muscular (Minúscoli, Meneghini, Oltramari, Wolpat, \& Melatti, 2013). As alterações sensoriais ocorrem em todos os sentidos, verificando-se uma perda maior de acuidade visual e de sensibilidade (Giro \& Paúl, 2013). Já as cognitivas envolvem todo o funcionamento mental, como a memória, a atenção, a aprendizagem e as alterações de linguagem (Vargas, Alves, Lara, \& Mello-Carpes, 2014).

Assim, percebe-se o quanto a motricidade fina é importante para os idosos, pois é uma atividade frequente e comum, caracterizada pela interação entre um objeto, o

*E-mail: michele.msilveira@gmail.com

- Submetido: 14/03/2016; Revisado: 07/08/2017; Aceito: 28/11/2017. 
olho e a mão. As habilidades motrizes estimuladas ajudam os idosos a conquistarem a sua independência no seu viver diário (Rosa Neto, 2009). A motricidade fina, em conjunto com a força muscular, é essencial para o processo do envelhecimento, seja para os membros inferiores presente na marcha, seja para os membros superiores na realização das atividades vitais (Fidelis, Patrizzi, \& Walsh, 2013). A diminuição da força muscular, em especial a preensão manual, pode gerar perdas na execução das atividades rotineiras para os idosos, como, por exemplo, abrir um pote de vidro com rosca, cortar um alimento com faca, utilizar o mouse do computador, entre outras.

Nessa perspectiva, Hollerweger, Almeida e Doll (2010) observaram que a habilidade para o uso do mouse por pessoas com idade superior a 45 anos depende de aspectos motores (motricidade fina), sensoriais (visão, tato), cognitivos (coordenação da mão - movimento do cursor) e motivacionais (medos e resistências). Já outros pesquisadores constataram que as tarefas de manipulação de objetos que exigem controle de força de preensão agem juntamente com o raciocínio mental (Voelcker-Rehage \& Alberts, 2007). Aplicando isso à presente pesquisa, percebese que esses fatores são valiosos para a interação correta com o computador, mas é visto que o desempenho motor da força de preensão manual e o desempenho cognitivo dos idosos têm sido estudados de maneira ostensiva e independentes um do outro, distanciando as ações da realidade.

Além disso, a manutenção de um desempenho cognitivo é fator considerável para a qualidade de vida e para o estado emocional na velhice (Batistoni et al., 2011). Um idoso com comprometimento cognitivo e com dificuldade para a execução das atividades da vida diária (AVDs) pode perder a independência, gerando incapacidades e diminuição da sua qualidade de vida (Giro \& Paúl, 2013).

Em vista disso, participar de grupos de convivência indica uma ação ativa na vida social, impactando muito positivamente na satisfação com a vida e no controle da saúde (Yassuda \& Silva, 2010). As oficinas de informática, que pertencem a grupos de convivência, proporcionam inúmeros benefícios aos idosos, evitam que se isolem, estimulam a sua memória, proporcionam novas amizades, promovem a comunicação e o entretenimento com um círculo de pessoas mais amplo (Silveira et al., 2011).

Dessa forma, considerou-se relevante verificar os efeitos do uso do computador no desempenho cognitivo, estado emocional (ansiedade e depressão), qualidade de vida e habilidade motora manual (força de preensão manual e motricidade fina) dos idosos que participaram das oficinas de informática, já que essa tecnologia está cada vez mais presente no cotidiano da maioria das pessoas.

\section{MÉTODO}

\section{Participantes}

A amostra deste estudo foi composta através da técnica de amostragem por conveniência por 114 sujeitos, dos quais 58 formaram o grupo sem informática (GSI) e 56 o grupo informática (GI). Para que os grupos fossem homogêneos com relação às variáveis sociodemográficas e em relação à atividade física, a participação dos idosos na pesquisa estava condicionada aos seguintes critérios: apresentar idade igual ou superior a sessenta anos; fazer parte de grupos de convivência; saber ler e escrever; não apresentar doenças neurodegenerativas avaliadas pelo exame cognitivo de Addenbrooke e nem doenças ortopédicas nos membros superiores (pós-operatório de cirurgia ortopédica, fratura recente) ou reumatológicas nas mãos (inflamação e deformidades) verificadas por questionários; para compor o GI, deveriam participar das oficinas de informática; para compor o GSI, não participar e nem ter participado de oficina de informática nos grupos de convivência.

O estudo observacional de corte foi realizado no município de Passo Fundo, localizado no interior do estado do Rio Grande do Sul, Brasil. Os dois grupos de convivência envolvidos na pesquisa foram: (a) Departamento de Atenção à Terceira Idade (Dati), incluso na Secretaria de Cidadania e Assistência Social (Semcas) da Prefeitura Municipal de Passo Fundo; (b) Centro Regional de Estudos e Atividades a Terceira Idade (Creati), da Universidade de Passo Fundo (UPF). Os indivíduos que participaram da pesquisa eram autônomos, socialmente ativos e residentes na comunidade. Para ambos os grupos, as aulas de informática ocorreram uma vez por semana, com duração de 1 h e $30 \mathrm{~min}$. O período de aulas de informática foi de seis meses, totalizando em torno de 24 aulas. O aluno que faltasse acima de $25 \%$ das aulas perdia a vaga na oficina.

\section{Instrumentos}

O questionário sociodemográfico incluiu variáveis como sexo, idade, estado civil, escolaridade, renda mensal, situação de moradia, ocupação atual, atividade física, oficinas que realiza no grupo de convivência, percepção de saúde, uso de medicação, patologias, percepção da memória, uso do computador.

O Exame Cognitivo de Addenbrooke (ACE-R) de Carvalho e Caramelli (2007) é um instrumento breve de rastreio cognitivo. As questões do instrumento são distribuídas nos seguintes domínios neurocognitivos: atenção e orientação (18 pontos), memória (26 pontos), fluência (14 pontos), linguagem ( 26 pontos) e visuo-espacial (16 pontos). Seus pontos de corte são calculados de acordo com a escolaridade: $\geq 50$ pontos para analfabetos; $\geq 60$ pontos para até quatro anos de estudo; $\geq 70$ pontos de cinco 
a oito anos de estudo e $\geq 80$ pontos mais que nove anos de estudo. Pontuações mais elevadas são indicativas de um melhor funcionamento cognitivo, atingindo um resultado máximo de 100 pontos.

A Escala de Depressão Geriátrica (GDS-15) utilizada identifica e quantifica sintomas depressivos em idosos, composta por 15 perguntas de respostas simples (sim ou não), o menor escore é zero e o maior é 15 . O valor é correspondente a um escore somatório maior que cinco pontos, que representam sintomatologia de depressão. Foi utilizada a versão validada do instrumento em português por Yesavage et al. (1983).

O Inventário de Ansiedade de Beck (BAI) mede a intensidade da ansiedade, constituído por 21 questões, na qual a resposta de cada questão varia de ausente, moderado, severo ou grave, com pontuação de zero a três, o maior escore possível é 63. Os sintomas de ansiedade correspondem a uma pontuação somatória maior que 20 pontos. Foi utilizada a versão traduzida e validada para a população brasileira da escala de Beck por Cunha (2001).

Já a escala de qualidade de vida (WHOQOL-Bref) foi desenvolvida pelo World Health Organization Quality of Life Group e validada para o português por Fleck et al. (2000), consta de 26 questões, das quais duas são gerais sobre qualidade de vida e 24 representam os domínios físico, psicológico, relações sociais e meio ambiente. Cada questão pode ser pontuada com valor de 1 a 5 . O instrumento não possui ponto de corte, quanto maior a pontuação, que varia de 0 a 100 , melhor a qualidade de vida.

Para avaliar a motricidade fina, utilizou-se o painel de habilidades manuais (Andreotti \& Okuma, 1999), formado por um painel retangular $(60 \mathrm{~cm}$ de comprimento, $15 \mathrm{~cm}$ de altura), que contém uma fechadura, uma tomada, um soquete para encaixar a lâmpada e um disco de telefone, dispostos horizontalmente a uma distância de $10 \mathrm{~cm}$ entre cada objeto. Para realizar o teste, é necessário um cronômetro, chave, plug e lâmpada. Iniciado o teste, o participante, em pé, em frente ao painel e com a mão dominante deve colocar a chave na fechadura, o plug na tomada, desencaixar a lâmpada do soquete e discar o número nove no disco do telefone com menor tempo possível. Para isso, são feitas duas tentativas, e a de menor tempo será registrada.

Para avaliar a força de preensão manual, utilizou-se o Dinamômetro Saehan Corporation ${ }^{\circledR}$, modelo SH5001, que é um instrumento válido e confiável (Reis \& Arantes, 2011). $\mathrm{Na}$ avaliação, leva-se em conta a mão dominante e a não dominante de cada sujeito. $O$ teste foi realizado na posição recomendada pela Sociedade Americana de Terapeutas de Mão: os testados ficaram sentados em uma cadeira sem braços, com os pés apoiados no chão e quadril e joelho posicionados a aproximadamente 90 graus de flexão. O ombro do membro testado ficou aduzido e em rotação neutra, cotovelo em flexão de 90 graus, antebraço na posição neutra e punho entre 0 e 30 graus de extensão e entre 0 a 15 graus de adução. A mão do membro não testado repousou sobre a coxa do mesmo lado.

Os participantes são instruídos a manter o posicionamento durante os testes e corrigidos pelo examinador quando necessário. O protocolo de testes foi dividido em duas partes: na primeira, os testes são realizados inicialmente com a mão dominante (direita) e depois com a mão não dominante (esquerda), de forma não alternada, visto que todos os participantes eram destros. Os participantes são instruídos a fazer uma contração máxima por três segundos em cada teste. Há um período de descanso de 30 segundos entre cada teste e um período de descanso de 2 minutos entre os testes de cada mão. É utilizada a média dos valores dos três testes de cada mão para a análise dos dados.

\section{Procedimentos}

Primeiramente, o projeto foi examinado e aprovado pela Comissão Científica do Instituto de Geriatria e Gerontologia. Em seguida, foi aprovado pelo Comitê de Ética em Pesquisa da PUCRS, Porto Alegre, RS, Brasil, parecer $n^{\circ} 241.858$. Após, foram realizados contatos com os grupos de idosos, procedendo, assim, à inclusão dos participantes na amostra. $\mathrm{O}$ presente estudo foi realizado em três etapas, as quais serão descritas a seguir. Ao todo, o tempo de coleta foi de dois anos e meio e o tempo utilizado para a aplicação pré e pós-atividade foi de seis meses, pois cada oficina de informática tinha a duração de seis meses. Nesse período total, ocorreram três inícios e três términos de avaliações.

$\mathrm{Na}$ Etapa 1, foi realizada uma entrevista individual, cada participante preencheu o termo de consentimento livre e esclarecido e respondeu sobre informações sociodemográficas e de saúde. Na sequência, foram avaliados o desempenho cognitivo (ACE-R), sintomas depressivos (GDS-15) e sintomas de ansiedade (BAI), após foram aplicados os testes de MF e FPM. Todos os instrumentos foram aplicados pelo mesmo avaliador, para uma padronização dos resultados, visto que os testes aplicados são objetivos e resultam em números. Para a avaliação cognitiva, o avaliador foi devidamente treinado, acompanhando por dez meses, uma vez por semana, as avaliações neuropsicológicas, que ocorriam no ambulatório de neuropsicologia do Hospital São Lucas da PUCRS. Inicialmente, todos os participantes das oficinas de informática foram avaliados, seguido dos pertencentes de outras oficinas nos grupos de convivência.

Nessa etapa, foram avaliados 174 indivíduos pertencentes a grupos de convivência, sendo excluídos do estudo 45 sujeitos, desses 25 por apresentarem idade inferior a sessenta anos, dois por uma pontuação muito inferior ao ponto de corte sugerido no ACE-R, três por não saberem ler e escrever e os 15 demais por apresentarem problemas nas mãos e dedos (patologias osteoarticulares e tremor).

A etapa 2 teve início com a participação de 129 idosos, 60 no GSI e 69 no GI. Como atividades realizadas nas oficinas 
de informática, os idosos que participavam não tinham conhecimento prévio do uso do computador e aprendiam a usá-lo na oficina, a realizar atividades como criar pastas, salvar documentos, digitar textos, salvar imagens, acessar e-mails, internet e redes sociais. O GSI envolveu participantes de outras oficinas de dança, alongamento, atividade física, entre outras, atividades que não exercitavam especificamente a motricidade fina, mas a motricidade ampla do indivíduo.

Dos 69 idosos do GI, 56 permaneceram efetivos durante o período dos seis meses de oficinas de informática. Foram realizadas em torno de 24 aulas, com $1 \mathrm{~h}$ e $30 \mathrm{~min}$ de uso do computador. As aulas foram coordenadas por professores de informática dos grupos de convivência. Dos 60 idosos do GSI, 58 permaneceram no período dos seis meses nos seus grupos de convivência. Os idosos que participaram do grupo GI estavam iniciando as atividades no computador, não haviam realizado oficinas de informática antes. Cada idoso tinha um computador individual, a maioria não tinha acesso ao computador em outro local e praticavam apenas no local das oficinas.

Na etapa 3, todos os participantes do GSI e do GI foram reavaliados imediatamente após o período dos seis meses de aulas de informática, observando o desempenho cognitivo, a depressão, ansiedade, qualidade de vida, motricidade fina e força de preensão manual. Os instrumentos foram os utilizados na etapa 1 desta pesquisa. Foram reavaliados 56 idosos do GI e 58 do GSI. Não foram reavaliados 15 idosos devido a problemas de saúde e por não estarem mais participando dos grupos. Assim, o número final de idosos incluídos nas análises foi de 114 .

\section{Análise dos Dados}

Os dados foram armazenados utilizando-se o MS Excel 2010 para Windows, posteriormente foram analisados utilizando o SPSS 17.0 para Windows. As variáveis numéricas foram descritas como média \pm desvio padrão ou mediana (p25 - p75), quando apresentaram distribuição marcadamente não normal. As variáveis categóricas foram expressas como frequência absoluta e relativa. A normalidade foi avaliada pela análise visual dos histogramas e pelo teste de Kolmogorov-Smirnov.

Para comparar as variáveis basais entre os grupos GI e GSI, utilizou-se o teste Anova como um critério de classificação para variáveis numéricas e qui-quadrado de Pearson para variáveis categóricas. Para avaliar o efeito do uso do computador sobre a cognição (ACE-R), os sintomas depressivos (GDS-15), ansiedade (BAI), qualidade de vida (WHOQOL-Bref), força de preensão manual (FPM) e motricidade fina (MF), ajustaram-se modelos de análise de variância, nos quais cada uma dessas variáveis (e os componentes do escore de cognição), pré e pós-atividade, foram especificadas como efeitos intraindivíduos (efeito de tempo), e o GI e GSI, como efeitos interindivíduos (efeito de grupo).

As médias marginais estimadas pelos modelos foram expressas com os respectivos intervalos de confiança de $95 \%$. Consideraram-se como estatisticamente significativos efeitos principais de tempo e de grupo com valor de probabilidade associado $<0,05$ e interações (tempo*grupo) com valor de probabilidade associado $<0,15$.

\section{RESULTADOS}

Participaram do estudo 114 idosos, 89 mulheres e 25 homens, com idade entre 60 e 83 anos, divididos em dois grupos: GSI, composto por 45 mulheres e 13 homens e GI, composto por 44 mulheres e 12 homens. A idade média de todos os participantes do estudo foi de $66,49 \pm 5,89$ anos (Tab. 1).

A tabela 1 descreve as características sociodemográficas da população em estudo. Os dois grupos foram formados, em sua maioria, por indivíduos casados, 28 no GSI (48,3\%) e 27 no GI $(48,2 \%)$ e 20 viúvos no GSI $(34,5 \%)$ e 16 no GI $16(28,6 \%)$. A escolaridade de ambos os grupos foi similar, com maior prevalência no GSI, de 11 anos de estudo, com vinte indivíduos $(34,5 \%)$ e 18 no GI $(32,1 \%)$. A renda mais presente dos grupos foi de um a dois salários mínimos: $58,6 \%$ no GSI e $53,6 \%$ no GI. A percepção de saúde predominante de ambos os grupos foi boa, a percepção de memória foi regular e a maioria revelou sentir-se esquecida.

Para avaliar o efeito do uso do computador, foi ajustado aos modelos de análise de variância, nos quais cada uma das variáveis, pré e pós-atividade, foram especificadas como efeito de tempo (o quanto variou ao longo do tempo - pré e pós-atividade), efeito de grupo (comparação entre os dois grupos - GSI e GI) e efeito tempo* grupo (mostra se o efeito do uso do computador foi estatisticamente significativo).

Quanto ao ACE-R total, observou-se que os efeitos tempo, grupo e interação tempo*grupo foram estatisticamente significativos, $p<0,001, p=0,020 \mathrm{e} p=0,008$, respectivamente. É possível visualizar, na Tabela 2, um ganho favorecendo apenas o GI.

Em relação ao GDS-15, observou-se que os efeitos tempo e interação tempo*grupo foram estatisticamente significativos, $\mathrm{p}=0,017 \mathrm{e} \mathrm{p}=0,110$, respectivamente, porém, no grupo, não foi estatisticamente significativo, $p=0,987$. $\mathrm{Na}$ Tabela 2, visualiza-se que ambos os grupos diminuíram a sintomatologia depressiva, porém o GI apresentou uma diminuição maior, estatisticamente significativa. Quanto ao BAI, observou-se que o efeito tempo $\mathrm{p}=0,011$, mas em interação tempo* grupo e grupo não foram estatisticamente significativos, $\mathrm{p}=0,810$ e $\mathrm{p}=0,837$, respectivamente. 
Tabela 1

Características Sociodemográficas da População em Estudo.

\begin{tabular}{|c|c|c|c|}
\hline \multicolumn{4}{|c|}{ Grupo } \\
\hline & $\begin{array}{c}\text { GSI } \\
(n=58)\end{array}$ & $\begin{array}{c}\text { GI } \\
(n=56)\end{array}$ & $\mathbf{P}$ \\
\hline Sexo & & & $0,899 *$ \\
\hline Masculino & $13(22,4)$ & $12(21,4 \%)$ & \\
\hline Feminino & $45(77,6 \%)$ & $44(78,6 \%)$ & \\
\hline Idade (anos) & $66,62 \pm 6,37$ & $66,35 \pm 5,39$ & $0,812 * *$ \\
\hline Faixa etária & & & $0,601 *$ \\
\hline $60-69$ anos & $42(72,4 \%)$ & $43(76,8 \%)$ & \\
\hline $70-79$ anos & $13(22,4 \%)$ & $12(21,4 \%)$ & \\
\hline $80-89$ anos & $3(5,2 \%)$ & $1(1,8 \%)$ & \\
\hline Estado civil & & & $0,641^{*}$ \\
\hline Solteiro (a) & $5(8,6 \%)$ & $4(7,1 \%)$ & \\
\hline Casado (a) & $28(48,3 \%)$ & $27(48,2 \%)$ & \\
\hline Separado (a) / Divorciado (a) & $5(8,6 \%)$ & $9(16,1 \%)$ & \\
\hline Viúvo (a) & $20(34,5 \%)$ & $16(28,6 \%)$ & \\
\hline Escolaridade & & & $0,776^{*}$ \\
\hline 4 anos de estudo & $17(29,3 \%)$ & $13(23,2 \%)$ & \\
\hline 8 anos de estudo & $7(12,1 \%)$ & $7(12,5 \%)$ & \\
\hline 11 anos de estudo & $20(34,5 \%)$ & $18(32,1 \%)$ & \\
\hline$\geq 12$ anos de estudo & $14(24,1 \%)$ & $18(32,1 \%)$ & \\
\hline Renda & & & $0,751^{*}$ \\
\hline 1-2 salários mínimos & $34(58,6 \%)$ & $30(53,6 \%)$ & \\
\hline 3-4 salários mínimos & $13(22,4 \%)$ & $16(28,6 \%)$ & \\
\hline$\geq 5$ salários mínimos & $11(19 \%)$ & $10(17,9 \%)$ & \\
\hline Percepção de saúde & & & $0,786^{*}$ \\
\hline Ótima & $9(15,5 \%)$ & $9(16,1 \%)$ & \\
\hline Boa & $28(48,3 \%)$ & $26(46,4 \%)$ & \\
\hline Regular & $20(34,5 \%)$ & $21(37,5 \%)$ & \\
\hline Ruim & $1(1,7 \%)$ & $0(0 \%)$ & \\
\hline Percepção de memória & & & $0,726^{*}$ \\
\hline Ótima & $1(1,7 \%)$ & $3(5,4 \%)$ & \\
\hline Boa & $25(43,1 \%)$ & $21(37,5 \%)$ & \\
\hline Regular & $27(46,6 \%)$ & $27(48,2 \%)$ & \\
\hline Ruim & $5(8,6 \%)$ & $5(8,9 \%)$ & \\
\hline Atividade física & & & $0,327^{*}$ \\
\hline Sim & $58(100 \%)$ & $50(89,3 \%)$ & \\
\hline Não & $0(0 \%)$ & $6(10,7 \%)$ & \\
\hline Sente-se esquecido & & & $0,935^{*}$ \\
\hline Sim & $49(84,5 \%)$ & $47(83,9 \%)$ & \\
\hline Não & $9(15,5 \%)$ & $9(16,1 \%)$ & \\
\hline
\end{tabular}

*Teste Qui-quadrado de Pearson.

**Teste Anova.

Na Tabela 3, observam-se os cinco domínios do ACE-R, o primeiro domínio, atenção e orientação, não foram observados, estatisticamente significativos, os efeitos tempo, grupo e interação tempo*grupo, $p=0,156, p=0,220 \mathrm{e}$ $\mathrm{p}=0,385$, respectivamente. No domínio memória do ACE-R, observou-se que os efeitos tempo e interação tempo*grupo foram estatisticamente significativos, $p=0,004, p=0,130$, respectivamente, mas não o efeito de grupo, $p=0,251$. Na Tabela 3, verifica-se ganho para ambos os grupos, maior no GI que no GSI. 
Tabela 2

Desempenho Cognitivo (ACE-R) e Estado Emocional (GDS-15 e BAI) entre os Grupos no Pré e Pós-Atividade.

\begin{tabular}{lccccc}
\hline \multirow{2}{*}{ Variáveis } & & \multicolumn{2}{c}{ Pré-atividade } & \multicolumn{2}{c}{ Pós-atividade } \\
\cline { 3 - 6 } & & Média & $\begin{array}{c}\text { IC95\% } \\
\text { Média }\end{array}$ & Média & $\begin{array}{c}\text { IC95\% } \\
\text { Média }\end{array}$ \\
\hline \multirow{2}{*}{ ACE-R } & GSI & 79,5 & $\begin{array}{c}73,1- \\
78,7\end{array}$ & 77,2 & $\begin{array}{c}74,4- \\
80,1\end{array}$ \\
& & & $76,3-$ & 83,2 & $80,3-$ \\
\multirow{2}{*}{ GDS-15 } & GI & 79,2 & 82,1 & 86,1 \\
& GSI & 3,4 & $2,8-3,9$ & 3,2 & $2,6-3,9$ \\
\multirow{2}{*}{ BAI } & GI & 3,6 & $3,0-4,2$ & 2,9 & $2,3-3,6$ \\
& GSI & 7,5 & $5,2-9,8$ & 6,2 & $4,1-8,2$ \\
& GI & 7,4 & $5,0-9,7$ & 5,7 & $3,7-7,8$ \\
\hline
\end{tabular}

Valores expressam média e respectivo intervalo de confiança de $95 \%$.

Quanto ao domínio fluência do ACE-R, observou-se que o efeito tempo, $\mathrm{p}=0,096$, o efeito grupo, $\mathrm{p}=0,338$, e interação tempo* grupo, $p=0,732$, não foram estatisticamente significativos. Com relação ao domínio linguagem do ACE-R (Tab. 3), observou-se que os efeitos tempo, grupo e interação tempo*grupo foram estatisticamente significativos, $\mathrm{p}<0,001$, $\mathrm{p}=0,002$ e $\mathrm{p}=0,006$, respectivamente, visualizando-se um ganho para ambos os grupos. Apesar de uma diferença basal favorecendo o GI, esse apresentou ganho maior que o GSI. Por fim, no domínio habilidades visuoespaciais do ACE-R (Tab. 3), observou-se que os efeitos tempo e grupo foram estatisticamente significativos, $p<0,001$ e $p<0,001$, respectivamente, mas não interação tempo*grupo, $\mathrm{p}=0,228$.

$\mathrm{Na}$ Tabela 4, observam-se os quatro domínios da escala de qualidade de vida WHOQOL-Bref. Com relação ao domínio físico do WHOQOL-Bref, observou-se que os efeitos tempo, grupo e interação tempo*grupo foram estatisticamente significativos, $\mathrm{p}=0,075, \mathrm{p}=0,075$ e $\mathrm{p}=0,120$, respectivamente. Observou-se um ganho para ambos os grupos, apesar de uma diferença basal favorecendo o GI, que apresentou ganho maior que o GSI, conforme demonstrado na Tabela 4.

Quanto ao domínio psicológico do WHOQOL-Bref, observou-se que os efeitos interação tempo*grupo, $\mathrm{p}=0,081$, tempo, $p=0,668$, e grupo, $p=0,187$, não foram estatisticamente significativos. Observou-se que os ganhos foram maiores no GI que o GSI. Já no domínio relações sociais do WHOQOLBref, observou-se que não houve efeito estatisticamente significativo para tempo, tempo*grupo e grupo, $\mathrm{p}=0,664$, $\mathrm{p}=0,664$ e $\mathrm{p}=0,994$, respectivamente. Os grupos diminuíram a pontuação desse domínio após o seguimento de seis meses de atividades.

Com relação ao domínio meio ambiente do WHOQOL-Bref, observou-se que o efeito grupo foi estatisticamente significativo, $\mathrm{p}=0,018$, mas não para os efeitos tempo e interação tempo*grupo, $\mathrm{p}=0,185$ e p $=0,075$, respectivamente. Observou-se um ganho para ambos,
Tabela 3

Domínios Cognitivos do ACE-R Pré e Pós-Atividade.

\begin{tabular}{|c|c|c|c|c|c|}
\hline \multirow[b]{2}{*}{ Variáveis } & & \multicolumn{2}{|c|}{ Pré-atividade } & \multicolumn{2}{|c|}{ Pós-atividade } \\
\hline & & Média & $\begin{array}{l}\text { IC95\% } \\
\text { Média }\end{array}$ & Média & $\begin{array}{l}\text { IC95\% } \\
\text { Média }\end{array}$ \\
\hline \multirow[t]{2}{*}{$\begin{array}{l}\text { Atenção } \\
\text { Orientação }\end{array}$} & GSI & 16,5 & $16,0-16,9$ & 16,6 & $16,2-17,0$ \\
\hline & GI & 16,8 & $16,3-17,2$ & 17,0 & $16,6-17,5$ \\
\hline \multirow[t]{2}{*}{ Memória } & GSI & 15,9 & $14,9-16,9$ & 16,3 & $15,2-17,4$ \\
\hline & GI & 16,2 & $15,2-17,2$ & 17,5 & $16,4-18,7$ \\
\hline \multirow[t]{2}{*}{ Fluência } & GSI & 9,1 & $8,4-9,7$ & 9,3 & $8,6-10,0$ \\
\hline & GI & 9,5 & $8,8-10,1$ & 9,8 & $9,1-10,5$ \\
\hline \multirow[t]{2}{*}{ Linguagem } & GSI & 21,6 & $20,7-22,4$ & 21,7 & $20,9-22,5$ \\
\hline & GI & 22,7 & $21,8-23,6$ & 23,9 & $23,0-24,7$ \\
\hline \multirow{2}{*}{$\begin{array}{l}\text { Habilidades } \\
\text { Visuo- } \\
\text { espaciais }\end{array}$} & GSI & 12,8 & $12,2-13,5$ & 13,3 & $12,7-13,9$ \\
\hline & GI & 14,0 & $13,3-14,6$ & 14,9 & $14,3-15,5$ \\
\hline
\end{tabular}

Valores expressam média e respectivo intervalo de confiança de 95\%.

apesar de uma diferença basal favorecendo o GI, que apresentou ganho um pouco maior que o GSI, conforme demonstrado na Tabela 4.

$\mathrm{Na}$ Tabela 5, observam-se as variáveis de força de preensão manual, como todos os participantes do estudo eram destros, a mão dominante foi a mão direita (FPM direita - FPMD), a mão não dominante foi a esquerda (FPM esquerda - FPME) e motricidade fina (MF). Quanto à FPMD, observaram-se estatisticamente significativos os efeitos tempo e interação tempo*grupo, $p=0,002$ e p $=0,035$, respectivamente, mas não o efeito grupo, $p=0,869$. $O$ GI teve um ganho maior que o GSI ao longo do tempo, demonstrando efeito significativo do uso do computador na FPMD.

Com relação à FPME, observaram-se estatisticamente significativos os efeitos tempo e interação tempo*grupo, $\mathrm{p}=0,002$ e $\mathrm{p}=0,079$, respectivamente, mas não o efeito grupo, $\mathrm{p}=0,726$. Quanto à MF, observaram-se estatisticamente significativos os efeitos tempo, interação tempo*grupo e grupo, $\mathrm{p}<0,001, \mathrm{p}=0,051 \mathrm{e} \mathrm{p}=0,029$, respectivamente. Observou-se um ganho para os grupos, apesar de uma diferença basal favorecendo o GI, que apresentou ganho maior que o GSI, ou seja, apresentou um melhor e mais rápido desempenho de motricidade fina, conforme demonstrado na tabela 5 .

Os valores visualizados na Tabela 5 demonstram melhores desempenhos de força de preensão manual e motricidade fina para ambos os grupos após a atividade com relação à pré-atividade, mas o GI apresentou valores de desempenho superiores e estatisticamente significativos no uso do computador. 
Tabela 4

Domínios de Qualidade de Vida entre os Grupos no Pré e Pós-Atividade.

\begin{tabular}{lccccc}
\hline Variáveis & & \multicolumn{2}{c}{ Pré-atividade } & \multicolumn{2}{c}{ Pós-atividade } \\
\cline { 3 - 6 } & & Média & IC95\% Média & Média & IC95\% Média \\
\hline Físico & GSI & 71,4 & $67,6-75,3$ & 71,7 & $68,0-75,3$ \\
& GI & 74,2 & $70,2-78,0$ & 77,8 & $74,1-81,5$ \\
Psicológico & GSI & 70,3 & $67,1-73,5$ & 69,1 & $65,6-72,6$ \\
& GI & 71,6 & $68,4-74,9$ & 73,7 & $70,1-77,2$ \\
Relações & GSI & 71,4 & $66,9-75,9$ & 69,1 & $64,8-73,4$ \\
Sociais & GI & 70,3 & $65,6-74,6$ & 70,2 & $65,8-74,6$ \\
\multirow{2}{*}{$\begin{array}{l}\text { Meio } \\
\text { Ambiente }\end{array}$} & GSI & 66,6 & $62,9-70,3$ & 67,1 & $63,1-71,2$ \\
& GI & 71,2 & $67,4-74,9$ & 74,8 & $70,7-78,9$ \\
\hline
\end{tabular}

Valores expressam média e respectivo intervalo de confiança de $95 \%$.
Tabela 5

Habilidade Motora Manual entre os Grupos no Pré e Pós-Atividade.

\begin{tabular}{lccccc}
\hline Variáveis & & \multicolumn{2}{c}{ Pré-atividade } & \multicolumn{2}{c}{ Pós-atividade } \\
\cline { 3 - 6 } & & Média & IC95\% Média & Média & IC95\% Média \\
\hline \multirow{2}{*}{ FPMD } & GSI & 24,3 & $22,4-26,2$ & 24,7 & $22,8-26,5$ \\
& GI & 23,3 & $21,4-25,3$ & 25,2 & $23,4-27,0$ \\
\multirow{2}{*}{ FPME } & GSI & 22,8 & $20,9-24,6$ & 23,3 & $21,5-25,0$ \\
& GI & 21,8 & $19,9-23,6$ & 23,4 & $21,7-25,2$ \\
\multirow{2}{*}{ MF } & GSI & 11,7 & $10,8-12,6$ & 10,6 & $9,8-11,4$ \\
& GI & 10,9 & $10,0-11,9$ & 8,8 & $8,0-9,7$ \\
\hline
\end{tabular}

FPM (força de preensão manual D - direita e E- esquerda). Valores expressam média e respectivo intervalo de confiança de $95 \%$.

\section{DISCUSSÃo}

Há poucos dados empíricos na literatura nacional e internacional sobre os efeitos das oficinas de informática na cognição, estado emocional, qualidade de vida e habilidade motora manual de idosos. Muitos estudos encontrados com idosos e informática preocupam-se mais em verificar o aprendizado, os benefícios e as dificuldades encontradas por eles na inclusão da tecnologia (Bolzan \& Lobler, 2013; Silveira et al., 2011; Silveira, Portuguez, Pasqualotti, \& Colussi, 2014). Outros verificam aspectos cognitivos de idosos que usam o computador (Ordonez, Yassuda, \& Cachioni, 2011; Scoralick-Lempke, Barbosa, \& Mota, 2012). Alguns ainda quantificam a qualidade de vida (Silveira et al. 2013), a ansiedade (Chu \& Mastel-Smith, 2010) e a depressão (Banhato et al., 2007) de idosos que participam de oficinas de informática. Poucas pesquisas buscam avaliar o desempenho motor do idoso que usa o computador (Medeiros et al., 2012).

Os resultados nos grupos quanto à interação do uso do computador, no seguimento de seis meses, demonstraram que o GI, comparado com o GSI, apresentou resultados significativos nos aspectos cognitivos, sintomas depressivos, qualidade de vida e habilidade motora manual. Com relação ao desempenho cognitivo, os resultados obtidos neste estudo mostram que os idosos do GI apresentam melhores escores no ACE-R pré e pós-atividade em todos os seus cinco domínios: atenção e orientação, memória, fluência, linguagem e habilidades visuoespaciais, apresentando diferença estatisticamente significativa quanto à interação na memória e linguagem.

Esses dados sugerem efeitos positivos no uso do computador no desempenho cognitivo de idosos e concordam com os estudos de Xavier, Sales, Ramos, Anção e Sigulem (2004), que realizaram oficinas de informática para idosos aposentados, buscando promover a inclusão digital com uma metodologia para a reabilitação cognitiva virtual e de Ordonez et al. (2011), que investigaram os efeitos de um programa de inclusão digital no desempenho cognitivo de 22 idosos que participaram de uma oficina de aprendizagem no uso do computador. Como efeitos, verificaram que o desempenho cognitivo do grupo experimental (GE) melhorou significativamente após o programa, em particular, nos domínios linguagem e memória do ACE-R e em seu escore total, quando comparados com o grupo de controle (GC), corroborando com os dados desta pesquisa.

O estudo de Small, Moody, Siddarth e Bookheimer (2009), envolvendo 24 indivíduos adultos e idosos, revelou que pesquisar na internet ativou de forma mais intensa o córtex pré-frontal, uma área que habilita o indivíduo a tomar decisões rapidamente ao avaliar informações complexas, sugerindo que aprender a usar um computador pode ser associado à neuroplasticidade. Scoralick-Lempke et al. (2012) revelam que a alfabetização digital de idosos serviu como um mecanismo de prevenção de déficit cognitivo nesses idosos saudáveis, que apresentavam uma média de escolaridade de nove anos e faixa etária entre 60 e 69 anos.

No presente estudo, verificou-se que os efeitos no uso do computador em relação aos sintomas depressivos foram estatisticamente significativos, mas não para a ansiedade. Ambos os grupos tiveram uma diminuição na média de pontuação das escalas de depressão e ansiedade, mas no GI a diminuição foi maior. Para Shapira, Barak e Gal (2007), os idosos que aprenderam a usar o computador e a internet mostraram uma melhora significativa nos aspectos psicológicos, como depressão, solidão e ansiedade. Os autores observaram que o uso da internet aumentou o bemestar e os sentimentos positivos relacionados à interação social, além de auxiliar no funcionamento cognitivo e preservação da autonomia e da independência.

Nesta pesquisa, verificou-se que os efeitos no uso do computador se estenderam para outras dimensões, ocorrendo 
uma melhora significativa no GI em relação à percepção de qualidade de vida no domínio físico. De maneira geral, os idosos do GI, no seguimento de seis meses, apresentaram aumento no seu desempenho cognitivo e melhor percepção de qualidade de vida em seus domínios quando comparados aos do GSI.

Estudos apresentaram uma associação entre desempenho cognitivo e qualidade de vida, evidenciando que quanto maior o declínio cognitivo, pior será a qualidade de vida dos idosos (Beckert, Irigaray, \& Trentini, 2012; Missotten et al. 2008). Para Ribeiro e Yassuda (2007), a capacidade cognitiva é um dos determinantes da qualidade de vida, pois perdas cognitivas podem resultar em comprometimento do funcionamento físico, social e emocional dos idosos.

Conforme Silveira et al. (2013), a qualidade de vida de idosos participantes de oficinas de informática com relação a não participantes demonstrou diferenças significativas no domínio físico, revelando boa disposição, mobilidade e capacidade funcional e, no domínio psicológico, comprovando sentimentos positivos em relação a aproveitar a vida. Nesta pesquisa, observou-se que o domínio físico foi o que mais apresentou diferença estatisticamente significativa, quando comparado com o grupo que não fez informática. Já no estudo de Heinz (2013), foi observado que a tecnologia e o computador proporcionaram melhor qualidade de vida e a independência dos idosos.

Além desses aspectos, pesquisas relacionadas ao envelhecimento e ao uso do computador demonstram que os idosos encontram alguns obstáculos no manuseio dessa tecnologia, referindo-se ao declínio das habilidades motoras, controle motor, atenção, memória e funções sensórias, como audição e visão. O declínio da motricidade fina, por exemplo, afeta a capacidade em usar o teclado e controlar o mouse. A diminuição da acuidade visual causa dificuldade em visualizar os ícones no monitor, bem como a acuidade auditiva diminuída dificulta todo o processo de ouvir as informações (Lindôso, Cammarota, Argimon, Gomes, \& Schwanke, 2011). Watanabe, Tsukimoto e Tsukimoto (2003) afirmam que as oficinas de informática para idosos promovem a capacidade de pensar, propõem melhorias nos aspectos motores nas atividades de vida diária (AVDs) e nas atividades instrumentais de vida diária (AIVDs). Atividades essas que necessitam de uma boa motricidade fina e força de preensão da mão para serem bem executadas.

Estudos que relacionaram a habilidade manual com o desempenho cognitivo revelam que a idade afeta, inevitavelmente, o aprendizado e o declínio das habilidades manuais dos idosos com maior idade, que mostraram um pior desempenho (Boyd, Vidoni, \& Siengsukon, 2008; Miyahara, 2007).

Com referência aos resultados encontrados nesta pesquisa, os efeitos do uso do computador na FPM e MF foram superiores no GI em relação ao GSI, ou seja, foram melhores no grupo que utilizou o computador, podendo ser justificado pelas atividades específicas de motricidade fina e força de preensão manual, mesmo que leves, que os idosos realizaram durante o período de informática para digitar e utilizar o mouse.

Há diversos tipos de pesquisas que avaliam a FPM em idosos, mas algumas a correlacionam com a flexibilidade (Fidelis et al., 2013; Silva, Menezes, Melo, \& Pedraza, 2013), outras revelam que a FPM é preditiva de sarcopenia (Pimentel \& Scheicher, 2013) e, ainda, existem estudos que verificam a sua relação com as atividades de vida diárias (Ribeiro \& Neri, 2012; Webber, Porter, \& Menec, 2010). A média dos valores de força de preensão manual dos idosos dos grupos GSI e GI desta pesquisa foram similares e correlacionaram a média de valores encontrados nos estudos de Macedo, Freitas e Scheicher (2014) e Sugiura et al. (2013), mas foram diferentes de Viveiro et al. (2014).

Com relação à motricidade fina, Lindôso et al. (2011) avaliaram as habilidades manuais de idosos que frequentavam oficinas de informática, cujos resultados permitiram observar que os idosos se saíram bem no teste com ambas as mãos, porém não apresentaram resultados estatisticamente significativos. Diferentemente deste estudo, obteve resultados significativos na motricidade fina, em que revelou que o computador é um instrumento de importante uso para a habilidade manual.

Katzer, Antes e Corazza (2012) utilizaram o mesmo instrumento deste estudo para avaliar a motricidade fina, porém a pesquisa foi com idosas praticantes e não praticantes de atividades físicas regulares, revelando que as praticantes obtiveram um desempenho superior $(7,72 \pm 1,79$ segundos) e significativo em relação às não praticantes $(9,28 \pm 2,51$ segundos). Já neste estudo, o GI obteve uma média de tempo 8,8 segundos e os do GSI 10,6 segundos, apresentando um desempenho pior que o encontrado no estudo de Katzer et al. (2012).

Em outro estudo com idosas praticantes de hidroginástica, observou-se que a idade influencia de forma significativa no desempenho da motricidade fina. Idosas com idade mais avançada tiveram um desempenho inferior em relação ao tempo de execução da tarefa, que foi 8 segundos para as de idade entre 59 e 69 anos e 9,09 segundos para as de 70-84 anos (Antes, Katzer \& Corazza, 2008). Pesquisadores (Pei et al., 2008) verificaram melhoras nos níveis de coordenação motora em idosos submetidos a um programa de atividade física generalizada de intensidade moderada, porém estudos que comparam a coordenação motora de idosos que usam o computador são pouco encontrados.

Lin et al. (2014) compararam a força de preensão manual e a coordenação bimanual das mãos de jovens e idosos e observaram que o envelhecimento reduz a produção de força de preensão manual e aumenta o tempo de desempenho do controle de coordenação bimanual das duas mãos, deixando-os mais lentos que os jovens, o que pode levar a 
dificuldades de execução de atividades diárias que exigem ambas as mãos.

Esses resultados corroboram com Meinel e Schnabel (1994), relatando que, em razão do processo de envelhecimento, há um comprometimento da coordenação motora e uma crescente diminuição do rendimento motor dos indivíduos idosos, percebendo regressões gradativas nas atividades diárias, profissionais ou psíquicas. Além disso, Cruz e Shirakawa (2006) avaliaram idosas praticantes de hidroginástica e não praticantes, verificando que as praticantes obtiveram resultados superiores quanto à motricidade fina e à estruturação espacial (propriocepção) em relação às não praticantes, ou seja, o grupo exposto à atividade de hidroginástica apresentou melhor desempenho motor manual.

Dessa forma, pode-se concluir que o uso do computador contribuiu para melhoras significativas no desempenho cognitivo total e nos seus domínios de memória e de linguagem, na sintomatologia de depressão, no domínio físico de qualidade de vida, na força de preensão manual e na motricidade fina, comparativamente ao GSI. Embora este estudo apresente resultados significativos quanto ao uso do computador, é necessário destacar a limitação encontrada que foi não ter uma avaliação cega dos idosos. Sugere-se que novos estudos sejam realizados com a avaliação cega dos sujeitos.

\section{REFERÊNCIAS}

Andreotti, R. A., \& Okuma, S. S. (1999). Validação de uma bateria de testes de atividades de vida diária para idosos fisicamente independentes. Revista Paulista de Educação Física, 13(1), 46-66.

Antes, D. L., Katzer, J. I., \& Corazza, S. T. (2008). Coordenação motora fina e propriocepção de idosas praticantes de hidroginástica. Revista Brasileira de Ciências do Envelhecimento Humano, 5(2), 24-32.

Argimon, I. I. L., \& Stein, L. M. (2005). Habilidades cognitivas em indivíduos muito idosos: Um estudo longitudinal. Cadernos de Saúde Pública, 21(1), 64-72.

Banhato, E. F. C., Silva, K. C. A., Magalhães, N. C., Mota, M. E., Guedes, D. V., \& Scoralick, N. N. (2007). Inclusão digital: Ferramenta de promoção de envelhecimento saudável? In Centro de Estudos em Psicologia da Saúde (Ed.), Anais do $4^{\circ}$ Congresso Interamericano de Psicologia da Saúde: Neurociências e Psicopatologia - Interfaces (pp. 45-46). São Paulo, SP: CEPSI.

Batistoni, S. S. T., Ordonez, T. N., Silva, T. B. L., Nascimento, P. P. P., Kissaki P. T., \& Cachioni1, M. (2011). Depressive symptoms in elderly participants of an open university for elderly. Dementia \& Neuropsychologia, 5(2), 85-92.

Beckert, M., Irigaray, T. Q., \& Trentini, C. M. (2012). Qualidade de vida, cognição e desempenho nas funções executivas de idosos. Estudos de Psicologia, 29(2), 155-162.

Bolzan, L. M., \& Löbler, M. L. (2013). As meninas estão na rede: A inclusão digital na terceira idade. Revista Espaço Pedagógico, 20(2), 301-312.

Boyd, L. A., Vidoni, E. D., \& Siengsukon, C. F. (2008). Multidimensional motor sequence learning is impaired in older but not younger or middle-aged adults. Physical Therapy, 88(3) 351-362.

Carvalho, V. A., \& Caramelli, P. (2007). Brazilian adaptation of the Addenbrooke's Cognitive Examination-Revised (ACE-R). Dementia \& Neuropsychologia, 1(2), 212-216.

Chu, A., \& Mastel-Smith, B. (2010). The outcomes of anxiety, confidence, and self-efficacy with Internet health information retrieval in older adults: A pilot study. Computers Informatics Nursing, 28(4), 222-228.

Cruz, C. C. P., \& Shirakawa, K. E. (2006). A relação da involução psicomotora com o número de quedas em idosas praticantes e não praticantes de exercício físico regular (hidroginástica) (Monografia). Recuperado de http://www.unama.br/ graduacao/fisioterapia/pdf/2006/a-relacao-da-involucaopsicomotora.pdf

Cunha, J. A. (2001). Manual da versão em português das escalas Beck. São Paulo, SP: Casa do Psicólogo.
Fechine, B. R., \& Trompieri, N. (2012). O processo de envelhecimento: as principais alterações que acontecem com o idoso com o passar dos anos. Inter science place, 1(20), 106-132.

Fidelis, L. T., Patrizzi, L. J., \& Walsh, I. A. P. (2013). Influência da prática de exercícios físicos sobre a flexibilidade, força muscular manual e mobilidade funcional em idosos. Revista Brasileira de Geriatria e Gerontologia, 16(1), 109-116.

Fleck, M. P. A., Louzada, S., Xavier, M., Chachamovich, E., Vieira, G., Santos, L., \& Pinzon, V. (2000). Aplicação da versão em português do instrumento abreviado de avaliação da qualidade de vida “WHOQOL-Bref". Revista de Saúde Pública, 34(2), 178-83.

Giro, A., \& Paúl, C. (2013). Envelhecimento sensorial, declínio cognitivo e qualidade de vida no idoso com demência. Actas de Gerontologia, 1(1), 1-10.

Heinz, M. (2013). Perceptions of technology among older adults. Journal of Gerontological Nursing, 39(1), 42-51.

Hollerweger, L., Almeida, S. T., \& Doll, J. (2010). Adultos maduros e informática: O mouse no caminho. Informática na Educação: Teoria \& Prática, 13(1), 167-179.

Katzer, J. I., Antes, D. L., \& Corazza, S. T. (2012). Coordenação motora de idosas. Conscientiae Saúde, 11(1), 159-163.

Lin, C.H., Chou, L.W., Wei, S.H., Lieuc, F.K., Chiangd, S.L., \& Sung, W.H. (2014). Influence of aging on bimanual coordination control. Experimental Gerontology, 53, 40-47.

Lindôso, Z. C. L., Cammarota1, M. P., Argimon, I. I. L., Gomes, I., \& Schwanke, C. H. A. (2011). Percepção subjetiva de memória e habilidade manual em idosos de uma oficina de inclusão digital. Revista Brasileira de Geriatria e Gerontologia, 14(2), 303-317.

Macedo, D. O., Freitas, L. M., \& Scheicher, M. E. (2014). Preensão palmar e mobilidade funcional em idosos com diferentes níveis de atividade física. Fisioterapia e Pesquisa, 21(2), 151-155.

Medeiros, F. L., Xavier, A. J., Schneider, I. J. C., Ramos, L. R., Sigulem, D., \& d'Orsi, E. (2012). Inclusão digital e capacidade funcional de idosos residentes em Florianópolis, Santa Catarina, Brasil (EpiFloripa 2009-2010). Revista Brasileira de Epidemiologia, 15(1), 106-122.

Meinel, K., \& Schnabel, G. (1994). Motricidade II: O desenvolvimento motor do ser humano. Rio de Janeiro, RJ: Ao Livro Técnico.

Minúscoli, A. V. F., Meneghini, G. O., Oltramari, J. D., Wolpat A., \& Melatti, J.(2013). Síndrome da Fragilidade: elucidando conceitos e propostas de avaliação e tratamento. In C. P. Dias \& J. C. F. Guimarães (Org.). Desvendando o envelhecimento (pp. 45-55). Caxias do Sul, RS: Faculdade da Serra Gaúcha. 
Missotten, P., Squelard, G., Ylieff, M., Di Notte, D., Paquay, L., De Lepeleire, J., ... Fontaine, O. (2008). Relationship between quality of life and cognitive decline in dementia. Dementia and Geriatric Cognitive Disorders, 25(6), 564-72.

Miyahara, M. (2007). Aging Influences on work memory for hand movements: a test of the metamemory deficit hypothesis. Experimental Aging Rasearch, 33(4), 417-28.

Ordonez, T. N., Yassuda, M. S., \& Cachioni, M. (2011). Elderly online: Effects of a digital inclusion program in cognitive performance. Archives of Gerontology and Geriatrics, 53(2), 216-219.

Pei, Y., Chou, S. W., Lin, P. S., Lin, Y. C., Hsu, T. H., \& Wong, A. M (2008). Eye-hand coordination of elderly people who practice Tai Chi Chuan. Journal of the Formosan Medical Association, 107(2), 103-110.

Pimentel, I., \& Scheicher, M. E. (2013). Comparação da mobilidade, força muscular e medo de cair em idosas caidoras e não caidoras. Revista Brasileira de Geriatria e Gerontologia, 16(2), 251-257.

Ribeiro, L. H. M., \& Neri, A. L. (2012). Exercícios físicos, força muscular e atividades de vida diária em mulheres idosas. Ciência \& Saúde Coletiva, 17(8), 2169-2180.

Ribeiro, P. C. C., \& Yassuda, M. S. (2007). Cognição, estilo de vida e qualidade de vida na velhice. In A. L. Neri (Org.), Qualidade de vida na velhice: Enfoque multidisciplinar. (pp. 189-204). Campinas, SP: Alínea.

Reis, M. M., \& Arantes, P. M. (2011). Medida da força de preensão manual - Validade e confiabilidade do dinamômetro Saehan Fisioterapia e Pesquisa, 18(2), 176-181.

Rosa Neto, F. (Org). (2009). Manual de avaliação motora para a terceira idade. Porto Alegre, RS: Artmed.

Scoralick-Lempke, N. N., Barbosa, A. J. G., \& Mota, M. M. P. E. (2012). Efeitos de um processo de alfabetização em informática na cognição de idosos. Psicologia: Reflexão e Crítica, 25(4), 774-782.

Shapira, N., Barak, A., \& Gal, I. (2007). Promoting older adults' well-being through Internet training and use. Journal of Mental Health and Aging, 11(5), 477-484.

Silva, N. A., Menezes, T, N., Melo, R. L. P., \& Pedraza, D. F. (2013). Força de preensão manual e flexibilidade e suas relações com variáveis antropométricas em idosos. Revista da Associação Médica Brasileira, 59(2), 128-135.

Silveira, M. M., Rocha, J. P., Kümpel, D. A., Wibelinger, L. M., Pasqualotti, A., \& Colussi, E. L. (2011). Ambientes de aprendizagem: Significado na vida de idosos frequentadores de oficinas de informática. Revista RENOTE: Novas Tecnologias na Educação, 9(1), 1-9.

Silveira, M. M., Tavares, G. M. S., Zuppa, C., Portuguez, M. W., Silva Filho, I. G., Carli, G. A., \& Colussi, E. L. (2013). Análise da qualidade de vida de idosos frequentadores de oficinas de informática. Conscientiae Saúde, 12(4), 598-603.

Silveira, M. M., Portuguez, M. W., Pasqualotti, A., \& Colussi, E. L. (2014). Envelhecimento e inclusão digital: Significado, sentimentos e conflitos. Revista Geriatria \& Gerontologia, $8,178-184$.

Small, G. W., Moody, T. D., Siddarth, P., \& Bookheimer, S. Y. (2009). Your brain on Google: patterns of cerebral activation during Internet searching. The American Journal of Geriatric Psychiatry, 17(2), 116-126.

Sugiura, Y., Tanimoto, Y., Watanabe, M., Tsuda, Y., Kimura, M., Kusabiraki, T., \& Kono, K. (2013). Handgrip strength as a predictor of higher-level competence decline among community-dwelling Japanese elderly in an urban area during a 4-year follow-up. Archives of Gerontology and Geriatrics, 57(3), 319-324.

Vargas L. S, Alves, N., Lara, M. V. S., \& Mello-Carpes, P. B. (2014). Conscientizando idosos e profissionais da saúde acerca das mudanças cognitivas relacionadas à idade. Revista Ciência em Extensão, 10(1), 37-50.

Viveiro, L. A. P., Almeida, A. S., Meira, D. M., Lavoura, P. H., Carmo, C. M., Silva, J. M., \& Tanaka, C. (2014). Declínio de atividades instrumentais de vida diária associado à perda de força de preensão palmar em idosos internados em enfermaria geriátrica. Revista Brasileira de Geriatria e Gerontologia, 17(2), 235-242.

Voelcker-Rehage, C., \& Alberts, J. L. (2007). Effect of motor pratice on dual-task performance in older adults. The Journals of Gerontology. Series B, Psychological Sciences and Social Sciences, 62(3), 141-148.

Watanabe, M., Tsukimoto, D. R., \& Tsukimoto, G. R. (2003). Terapia ocupacional e o uso do computador como recurso terapêutico. Acta Fisiátrica, 10(1), 17-20

Webber, S. C., Porter, M. M., \& Menec, V. H. (2010). Mobility in older adults: A comprehensive framework. The Gerontologist, 50(4), 443-450.

Xavier, A. J., Sales, M., Ramos, L., Anção, M., \& Sigulem, D. (2004). Cognition, interaction and ageing: An Internet workshops exploratory study. In L. Bos, S. Laxminarayan, \& A. Marsh (Eds.), Medical and Care Compunetics 1 (Studies in Health Technology and Informatics, Vol. 103, pp. 289-295). Amsterdam: IOS Press.

Yassuda, M. S., \& Silva, H. S. (2010). Participação em programas para a terceira idade: impacto sobre a cognição, humor e satisfação com a vida. Estudos de Psicologia, 27(2), 207-214.

Yesavage, J., Brink, T. L., Rose, T. L., Lum, O., Huang, V., Adey, M., \& Leirer, V. O. (1982/1983). Development and validation of a geriatric depression screening scale: a preliminary report. Jounal of Psychiatry Research, 17(1), 37-49. 